einen Blatt. Doch wird damit auch jede fachübergreifende Berufsausübungsgemeinschaft automatisch unter den Verdacht der Strafbarkeit gestellt? Was ist mit dem Kollegen in der BAG, der, anders als die anderen, über die Abrechnungsgenehmigung für bestimmte Laborleistungen verfügt? Nach der Gesetzesbegründung fallen unter den Begriff der Zuführung von Patienten auch solche im Rahmen vertraglicher Kooperationen wie eine Berufsausübungsgemeinschaft. Unter $\mathrm{Zu}$ führung von Untersuchungsmaterial ist insbesondere die Weiterleitung von Proben zur Durchführung von Laboruntersuchungen, aber auch zur Histologie gemeint. Und diese kann auch in einer Berufsausübungsgemeinschaft stattfinden.

Der Wortlaut der neuen Strafnormen ist weit, die Gesetzesbegründung kann als Interpretationshilfe herangezogen werden, hilft aber oft nicht weiter. Es wird jedoch eine Weile dauern, bis sich hierzu in der Rechtsprechung klare Linien herausgebildet haben. Hier sind Ärztekammern und Juristen gefragt, um die Grenzen einer unlauteren und einer lauteren Kooperation zu definieren.

Für die Vertragspraxis heißt dies, dass bei jeglicher Vertragsgestaltung - beginnend bei Arbeits- und Gesellschaftsverträgen bis hin zu Honorararzt, Belegarzt und jeglichen Kooperationen im Gesundheitswesen - genau zu prüfen ist, welche Regelungsinhalte den gesetzlichen Vorgaben entsprechen. Dies gilt auch und insbesondere für das ärztliche Engagement in Unternehmen im Gesundheitswesen, die - z. B. wie eine Physiotherapiepraxis- die ärztliche Leistungserbringung sinnvoll ergänzen. Auch bestehende vertragliche Beziehungen sollten überprüft werden, denn was noch vor Kurzem als zulässig erachtet wurde, kann gegen die neuen Regelungen im Strafgesetzbuch verstoßen.
Bereits der Verdacht der Korruption und Einleitung eines strafrechtlichen Ermittlungsverfahrens kann für die Betroffenen $\mathrm{zu}$ einem nicht wieder gutzumachenden Schaden führen - dies selbst dann, wenn am Ende nichts dabei herauskommt. Es liegt also im Interesse aller Akteure im Gesundheitsmarkt, bestehende oder zukünftige Kooperationen auf Herz und Nieren zu prüfen.

\section{Sozietät Hartmannsgruber Gemke Argyrakis \& Partner Rechtsanwälte Rechtsanwälte und Fachanwälte für Medizinrecht Dr. Gwendolyn Gemke}

August-Exter-Straße 4

81245 München

www.med-recht.de

\title{
Marketing - es ist mehr erlaubt als gedacht
}

\section{Verboten ist Werbung heute dann, wenn sie irreführend, vergleichend oder anpreisend ist. Ansonsten gibt es viel Spielraum für gute Ideen auch im Bereich der sozialen Medien.}

\begin{abstract}
Vom früheren Werbeverbot für Ärzte ist in der Berufsordnung und im Heilmittelwerbegesetz nicht mehr viel übrig geblieben. Vieles, was früher verboten war, ist heute erlaubt - und das gilt auch für Marketing in sozialen Medien. Rechtsanwalt Professor Thomas Schlegel machte Ärzten und Zahnärzten bei einem Praxisseminar der Deutschen Apotheker- und Ärztebank (apoBank) in Frankfurt daher Mut, ,auch mal etwas auszuprobieren und die Möglichkeiten auszuloten“.
\end{abstract}

\section{Video auf YouTube ...}

Schlegel regte an, sich auch bei den sozialen Medien kundig zu machen, um sie dann für eigene Aktivitäten zu nutzen. Beispiel YouTube, „die zweitgrößte Suchmaschine weltweit", wie Schlegel erläuterte. „Gehen Sie mal drauf und sehen Sie, was die Leute alles als Video machen, zum Beispiel Image-Videos oder auch Erläuterungen einer Therapie." Für Fachärzte könne es sich lohnen, Videos zur Unterstützung der Aufklärung bei YouTube ins Netz zu stellen. Das sei zwar kein Ersatz für tatsächliche Aufklärung, es könne den Aufklärungsprozess in der Praxis aber beschleunigen, wenn der Patient bereits vorinformiert ist.

Schlegel empfahl den Zuhörern auch regelmäßig im Web zu suchen, was dort über sie berichtet wird, besonders in den wichtigsten Bewertungsportalen wie jameda.de. Bei schlechten Beurteilungen sei es manchmal sinnvoll, online persönlich Stellung zu nehmen. Aktives Bewertungsmarketing etwa über Bewertungsbögen direkt am Empfang der Praxis könne dabei helfen, auf den einschlägi-
Es kann sich lohnen und ist machbar, Videos zur Unterstützung der Patientenaufklärung beispielsweise bei YouTube ins Netz zu stellen.

gen Portalen gut benotet zu werden und bei einer schlechten Kritik nicht gleich in der Durchschnittsnote abzustürzen.

\section{... oder auch der gute alte Brief}

Sehr sinnvolle Dienste könne auch im Zeitalter von Social Media immer noch die E-Mail oder sogar ein Brief auf Papier leisten, etwa bei einer Praxisübernahme, betonte Schlegel. „Allein dürfen Sie die Patienten des abgebenden Arztes nicht anschreiben, aber in einem gemeinsamen Brief ist das schon möglich, dafür dürfen Sie die Adressen nutzen“, erläuterte der Rechtsanwalt. In dem Schreiben könne der Verkäufer-Arzt darum werben, dass die Patienten dem Käufer ihr Vertrauen weiterhin schenken. Schlegel empfahl, ein solches gemeinsames Patientenschreiben mit in den Kaufvertrag aufzunehmen. Hauke Gerlof 